\title{
Effects of Fading Channels on OFDM
}

\author{
Ahmed Alshammari, Saleh Albdran, and Dr. Mohammad Matin \\ Department of Electrical and Computer Engineering University of Denver
}

\begin{abstract}
One of the essential components of recent communications is Orthogonal Frequency Division Multiplexing (OFDM). Handling bad conditions, high bandwidth and using the available spectral efficiently are some of its characteristics. Hence, it has replaced old communication technologies in many systems such as wireless networks and $4 G$ mobile communications. In this paper, Effects of fading channels on OFDM are investigated. MATLAB is used to simulate wireless fading channels environments that are either based on Doppler spread or Delay Spread.
\end{abstract}

Key Words-OFDM, ISI, BER, PSNRS

\section{INTRODUCTION}

High data rates are demanded a lot in modern communications. It is the huge development in the communications industry that led to this demand. Also, better quality and lower BER became more important. OFDM technique, which was introduced in the 1960's, provides all that [1][2]. It was not practical at that time because technologies to apply did not exist then e.g. it was not possible to have processors that can perform IFFT and FFT. In the 90's many of those problems were solved and OFDM started getting more popular since then.

Multi carrier modulation is the backbone of the OFDM technique. Hence, data are split to many parallel streams, which decreases bit rate. OFDM system of modulates several subcarriers using these parallel sub streams. OFDM's ability to transmit data with high speed is the main reason it is getting so popular besides robustness against Inter symbol interference (ISI). Therefore, many wireless and wired communication standards around the world have adopted this kind of modulation.

OFDM system model used in this paper is shown in Figure 1. Series to parallel conversion of the data stream results in the blocks of data symbols that we are transmitting. Assuming that $\mathrm{N}$ is the number of these blocks then OFDM symbols are characterized by $X_{m}=\left(x_{0, m} x_{1, m} \ldots x_{N-1, m}\right)^{T}$. After that each one of the symbols modulates one subcarrier. A guard band of length $N_{c p}$ is attached to each OFDM symbol. The $m^{\text {th }}$ OFDM symbol is given by equation (1).

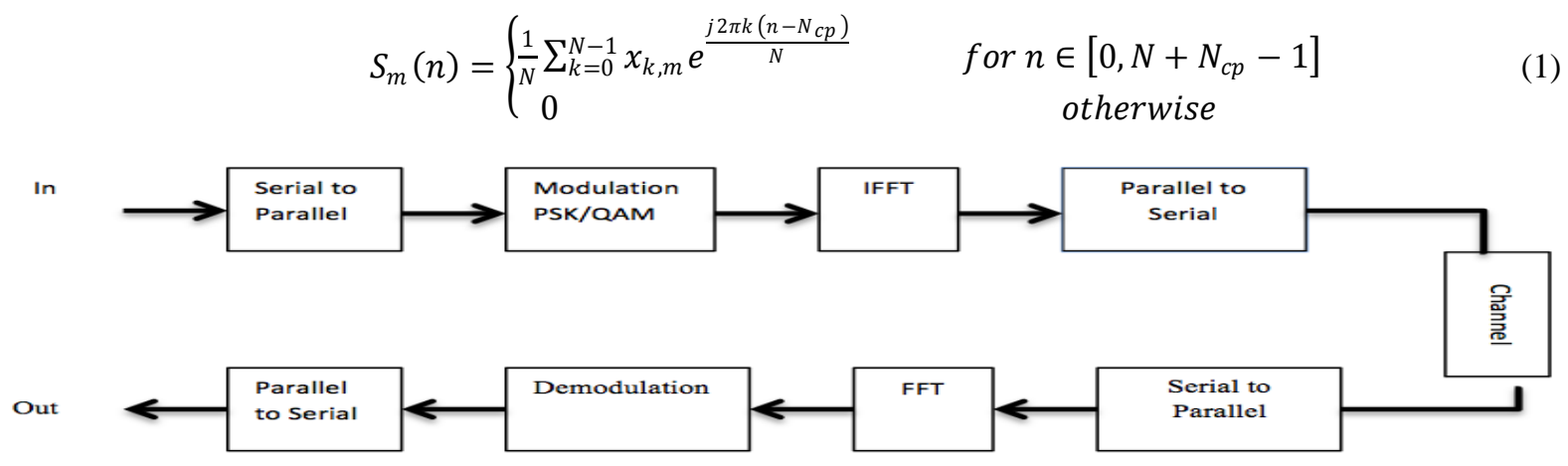

Figure 1 Block diagram of the OFDM system

Equation (2) represents the received signal $r(n)$ [3]. We can notice that the received signal is the summations of the white Gaussian noise (AWGN) $n(n)$ and the convolution of the channel's impulse response $h(n)$.

$$
r(n)=\sum_{\eta}^{n_{c p}-1} h(\eta) s(n-\eta)+n(n)
$$

\section{FADING}

Communication channel is the medium that connects the transmitter and the receiver. Channels could vary according to the nature of the system. The loss in the signal as it approaches the receiving side is caused by 
random attenuations. In wireless environment, it is possible that signals propagate through two or more paths before reaching the receiver in a phenomenon that is known as multipath propagation. There are many reasons that can cause multipath such as atmospheric conditions or lack of direct bath. Phase shifting, destructive and constructive interference are some of the consequences of this phenomenon [4]. Hence, received signal could have different amplitude and phase because of change in the propagation time and the intensity distribution of the waves [5].

\subsection{Small Scale Fading}

Small scale fading is controlled by the nature of the sent signal and communication environments. Symbol duration, Bandwidth and channel parameter are factors that decide the type of fading channel. Small scale fading could be divided into two main categories as shown in figure 2:

1) Fading Due To Delay Spread: Delay spread can cause two types fading that are either frequency selective slow and frequency selective fast fading [5]. Flat fading is the most popular types of fading. It occurs when the bandwidth of the signal is less than the bandwidth of the channel. The power of the signal is reduced in this case as a consequence to the gain variation of the channel when the spectrum remains the same. In frequency selective fading: linear phase response and constant gain are the main characteristics. Bandwidth of the channel is smaller than that of the signal, which is distorted by frequency selective, fading due to the multiple version of the signal with various amplitudes that are received.

2) Fading From Doppler Spread: it is either flat slow or flat fast fading caused by Doppler spread. In slow fading, symbol period is more than coherence. In Fast Fading, impulse response of the channel variations are very fast i.e. symbol duration of the transmitted signal is more than the coherence time.

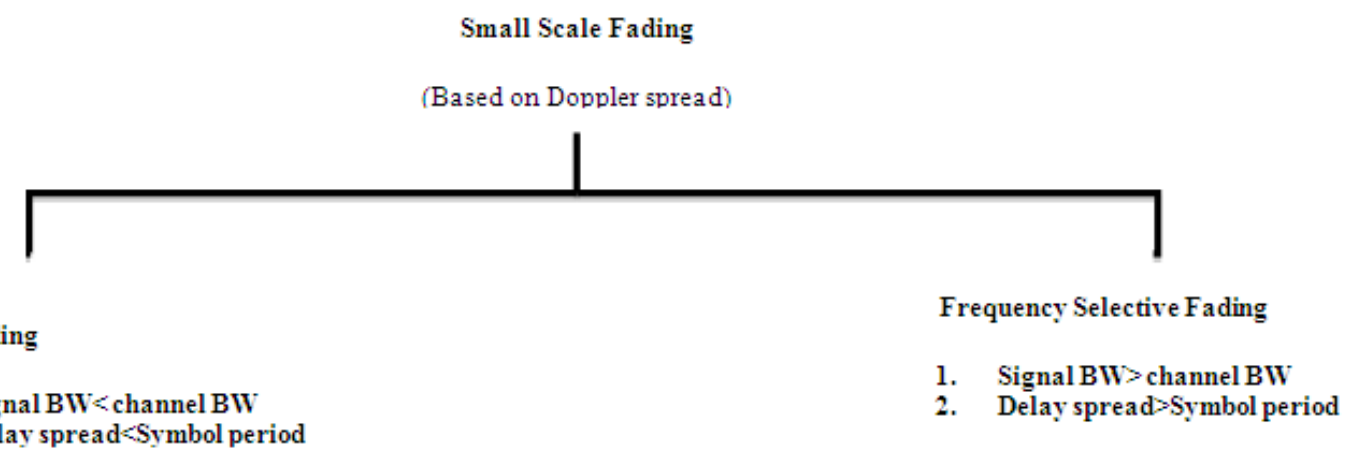

1. Signal $B W<$ channel BW

Small Scale Fading

(Based on multipath delay spread)

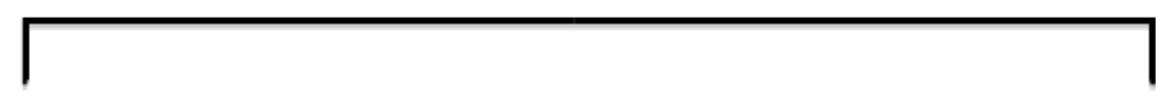

Fast fading

1. High Doppler Spread

2. Coherence time $<$ Symbol period

3. Channel changes faster than base band signal changes

\author{
Slow Fading \\ 1. Low Doppler Spread \\ 2. Coherence time $>$ Symbol period \\ 3. Channel changes Slower than base \\ band signal changes
}

Figure 2 Small Scale Fading

\subsection{Factors affecting fading:}

Four things could affect small scale fading:

1) Mobile speed: moving mobile could experience positive or negative Doppler shift. When the mobile moves closer to the transmitting side positive Doppler spread happened while Negative Doppler spread occurs the other way around.

2) Multipath Propagation: Signal energy is consumed by phase, amplitude and time in multipath environment. Multiple versions of the signal with various arriving instants and shifted spatial orientation. The duration that the signal takes to get to the receiver is increased in multipath channels.

3) Bandwidth of The Channel: When the bandwidth of the channel is less that that of the signal transmitted, it gets distorted. The channel transfer function must be flat in order for its bandwidth to be coherent. Transfer function of the channel is flat whenever the phase response is linear with a constant gain [6]. 
4) Velocity of objects within the channel: Objects in the communication environment sometimes are moving. If that movement is of a speed that is more than that of the receiver, small-scale fading happens otherwise we can ignore those movements.

\section{CHANNEL MODELING}

Time variant impulse response of the channel is used to model it. Motion of the receiver is the variable that affects time changing. Equation (3) is calculates the received signal.

$$
y(d, t)=\int_{-\infty}^{t} x(\tau) h(d, t-\tau) d(t)
$$

Where $h(d, t)$ is the impulse response of the channel and $x(t)$ is the signal. If $d=v t$ is the position of the receiver and $v$ is a constant, it is possible to replace $d$ in equation (3) with $v t$ as in equation (4).

$$
\begin{aligned}
y(v t, t) & =\int_{-\infty}^{t} x(\tau) h(v t, t-\tau) d(t) \\
h_{b}(t, \tau) & =\sum_{i=0}^{N-1} a_{i}(t, \tau) e^{\left[j\left(2 \pi f_{c} \tau_{i}(t)+\varphi_{i}(t, \tau)\right)\right]} \delta\left(\tau-\tau_{i}(t)\right)
\end{aligned}
$$

Channel impulse response can be found using (5). Where, $a_{i}(t, \tau)$ are the amplitudes and $\tau_{i}(t)$ are the delays whereas $\theta_{i}(t, \tau)=2 \pi f_{c} \tau_{i}(t)+\varphi_{i}(t, \tau)$ represent phase shift in the $i$ th component.

The impulse response can be found using equation (9) for time invariant channel where every multipath components of that cannel have delay.

$$
h_{b}(\tau)=\sum_{i=0}^{N-1} a_{i}(t, \tau) e^{\left[j \theta_{i}(t, \tau)\right]} \delta\left(\tau-\tau_{i}(t)\right)
$$

\subsection{Clarke's Model}

Clarke's Model relies on scattering to discover the statistical characteristics of the channel. Beside the assumption of fixed transmission antenna that is vertically polarized, $\mathrm{N}$ normalized plane waves of the antenna with a random carrier phases angels of arrival are assumed while amplitude remains unchanged. When the receiver is moving, $m_{t h}$ wave that has arriving angle $\alpha_{m}$ with respect to the x-axis. Doppler shift is given by equation (10).

Where, $\lambda$ is the wavelength of incident wave.

$$
f_{m}=\frac{v}{\lambda} \cos \alpha_{m}
$$

Every received wave has a different carrier frequency with small shift from the center frequency. The power spectral density of the output is given by equation 11. It is clear from that equation that the PDF is zero when $\left|f-f_{c}\right|>f_{m}$. With center frequency $f_{c}$, Spectrum is limited to $\pm f_{m}$ and all the received waves has different carrier frequencies that are shifted.

$$
S(f)=\frac{A[q(\alpha) G(\alpha)+(-\alpha) G(-\alpha)}{f_{n} \sqrt{1-\left(\frac{f-f_{c}}{f_{m}}\right)^{2}}}
$$

Assuming a vertical $\frac{\lambda}{4}$ antenna, $G(\alpha)=1.5$ and $p(\alpha)=\frac{1}{2 \pi}$ over 0 to $180^{\circ}$, then $S(f)$ becomes as in equation (12).

$$
S(f)=\frac{1.5}{\pi f_{m} \sqrt{1-\left(\frac{f-f_{c}}{f_{m}}\right)^{2}}}
$$

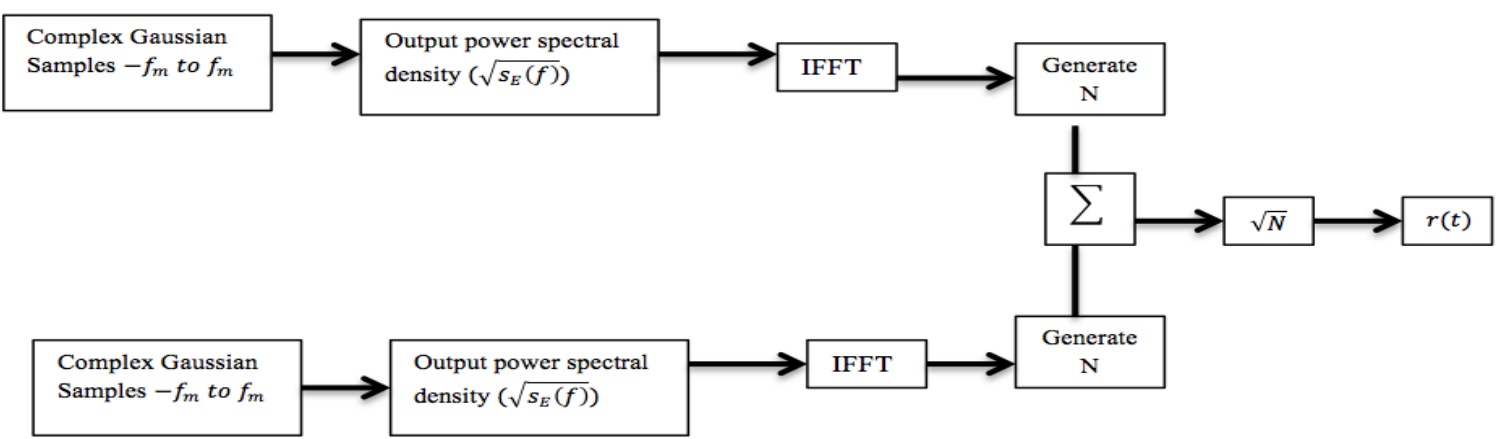

Figure 3 Rayleigh fading implementation 
The block diagram in figure 3 was used to simulate Rayleigh fading in the frequency domain. First, The signal is modulated with In-phase \& quadrature modulation. Thus, two independent Gaussian low pass noise components are used to create these components. IFFT is implemented as the last step in the simulation process in order to shape the random signal.

The simulator in figure 3 is impended according to the following steps [5]:

- $\quad$ Determine the number of points in frequency domain $\mathrm{N}$ which describes the square root of PDF $\sqrt{S_{E_{z}}(f)}$ and the max Doppler frequency shift $f_{m}$ should be specified as well.

- Find out the time duration of the fading waveform that is given by $T=\frac{1}{\Delta f}$.

- Create complex Gaussian Radom variables for each $\frac{N}{2}$ positive frequency components.

- Conjugate positive frequencies in order to find the negative ones.

- Multiply the in-phase and quadrature components by fading spectrum $\sqrt{S_{E_{z}}(f)}$

- Apply IFFT on the in-phase and quadrature components in order to obtain two N times series. Sum the squares of each signal point.

- Take the square foot of the summation done in the previous step in order to obtain the N Pint time series with the Doppler spread and the time correlation for the Rayleigh fading.

\section{RESULTS}

OFDM technique is studied over wireless communication environment to examine the effects of fading. MATLAB to simulate the process of transmitting image signals over fading channels with various SNRs. OFDM model discussed in the first part of this paper was followed and Fading channels were implemented according to Clarke's model. It assumes that there is no line of sight between transmitter and receiver ends. For flat and frequency selective fading, the speed of the receiver was either slow 3-miles/ hour or fast 100-miles/hour. 3 Miles/hour was assigned as the speed of the receiver for the purpose of simulating Small Doppler Spread and 100 Miles/hour was used to simulate large Doppler Spread. Figures 4 and 5 shows images sent over Flat slow Fading and Flat Fast Fading channels respectively with different SNRs. It is clear that quality of the received signals is improved at higher SNRs. Also, images have more distractions at higher speeds of the receiver. Therefore, the OFDM system delivers better images than over flat slow fading.

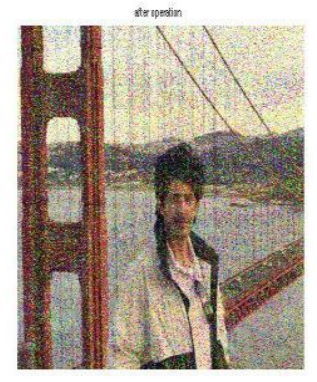

$0 \mathrm{~dB}$

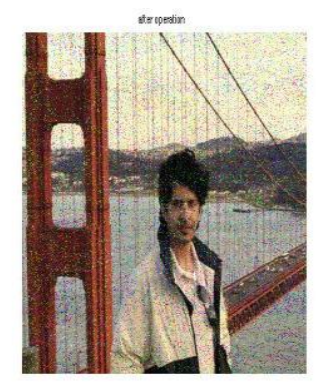

$4 \mathrm{~dB}$

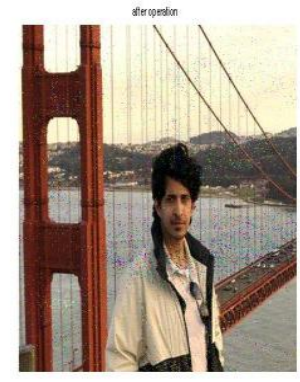

$8 \mathrm{~dB}$

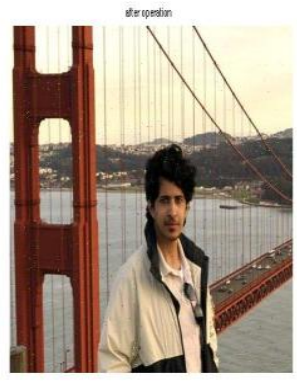

$12 \mathrm{~dB}$

Figure 4 Image sent Over Flat Slow Fading Channel with Different SNRs

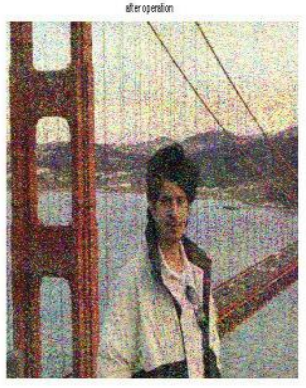

OdB

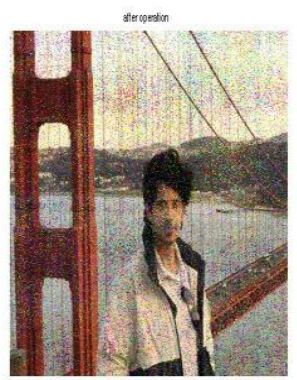

$4 \mathrm{~dB}$

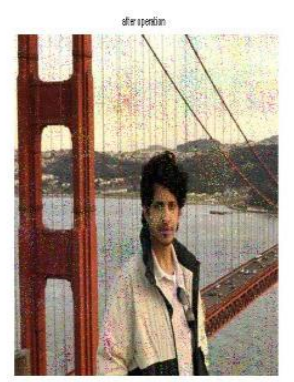

8dB

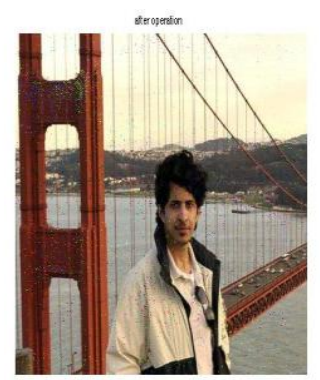

$12 \mathrm{~dB}$

Figure 5 Image sent Over Flat Fast Fading Channel with Different SNRs 
For Frequency selective fading environment, Multiple Clarke's model was used. Multiple delayed versions of the transmitter signal are subjected to Clark's model to simulate frequency selective Fading. 4 different paths are assumed with delay of $0,4,8$ and 16 samples. As done in flat fading, 3 Mile/hour mobile speeds was used in the simulation for frequency selective slow fading and $100 \mathrm{Mile} / \mathrm{hour}$ speed was used to simulate Frequency Selective Fast Fading. Figures 6 and 7 shows BER over Frequency Selective Slow Fading and Frequency Selective Fast Fading respectively.

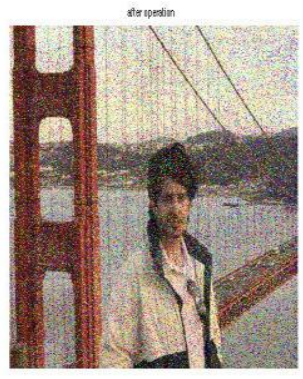

OdB

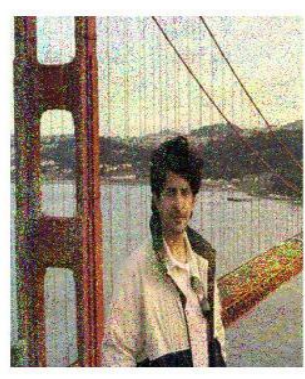

$4 \mathrm{~dB}$

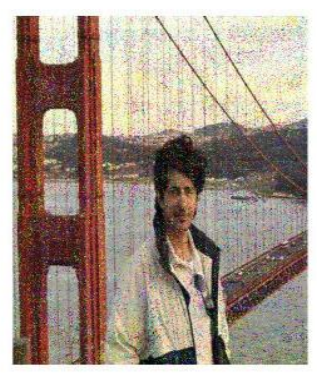

$8 \mathrm{~dB}$

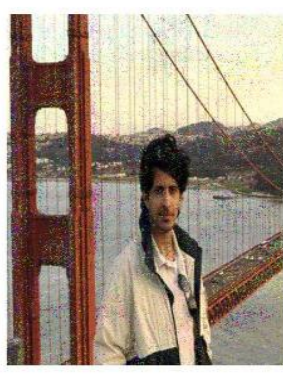

$12 \mathrm{~dB}$

Figure 6 Image sent Over Frequency Selective Slow Fading Channel with Different SNRs

Similarly, Quality of the received images is improved at higher SNRs for frequency selective fading channel. However, images have more distractions at high receiver speeds. Therefore, OFDM system delivers better images over Frequency Selective slow fading than frequency selective fast fading when the speed of the receiver is increased.

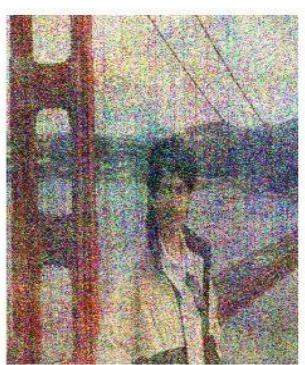

OdB

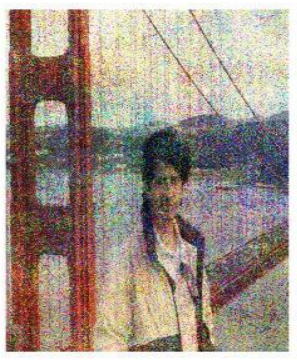

$4 d B$

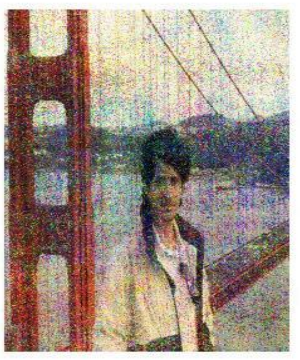

$8 \mathrm{~dB}$

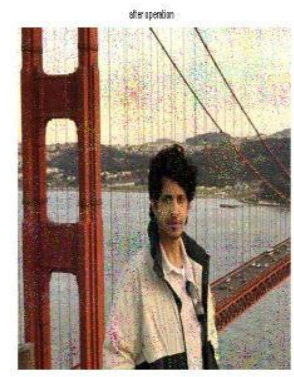

$12 \mathrm{~dB}$

Figure 7 Image sent Over Frequency Selective Fast Fading Channel with Different SNRs

OFDM system shows a better performance over flat fading than frequency selective fading. Also, the quality of the received signal is less when the mobile is moving with higher speeds. BER of the four kinds of Fading are shown in figure 8 . It is clear that the signal received in frequency selective fading channel environment has more BER than the one that is received in flat fading channel environment.

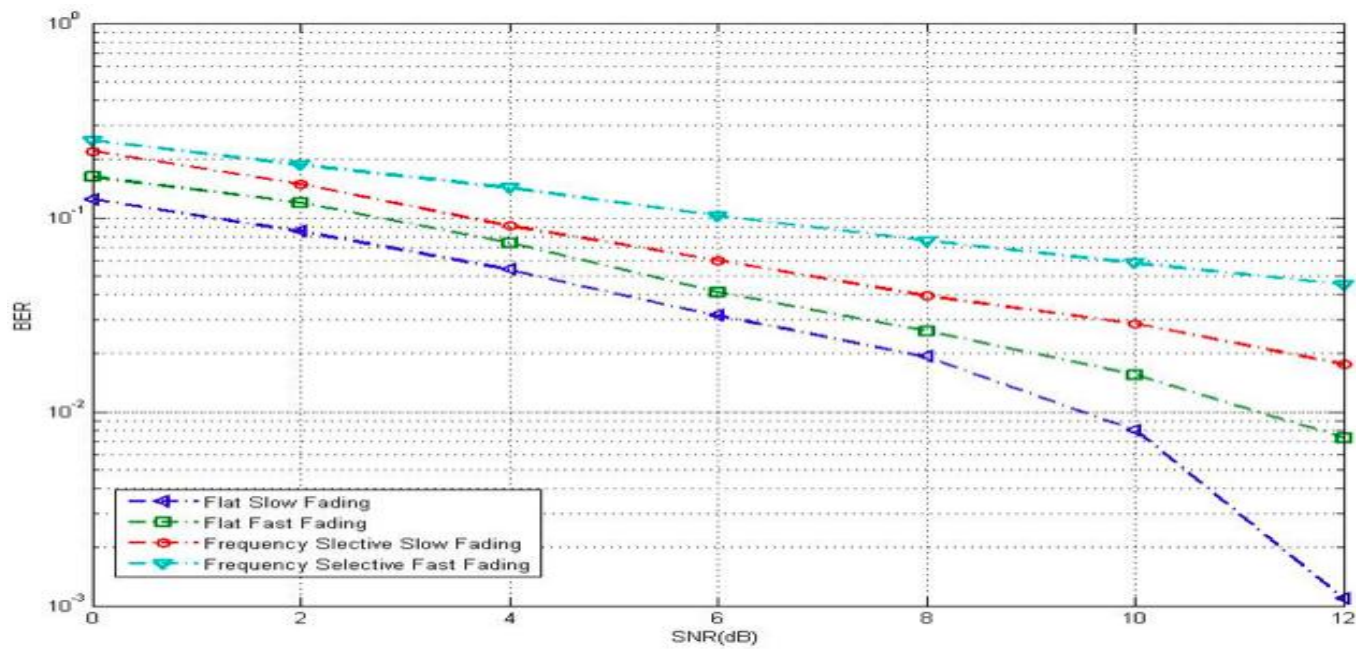

Figure 8 Comparing BER of The Different Channels 
Peak-Signal-to-Noise Ratio (PSNR) is used to measure reconstruction quality of the received signal. It is simply the ratio between the power of the original signal and the power of the disturbing noise that affect the quality of the received signal. PSNRs curves for the received images are shown in figure 9. We can see the OFDM System delivers images with more PSNRs flat fading channel than in frequency selective fading channel.

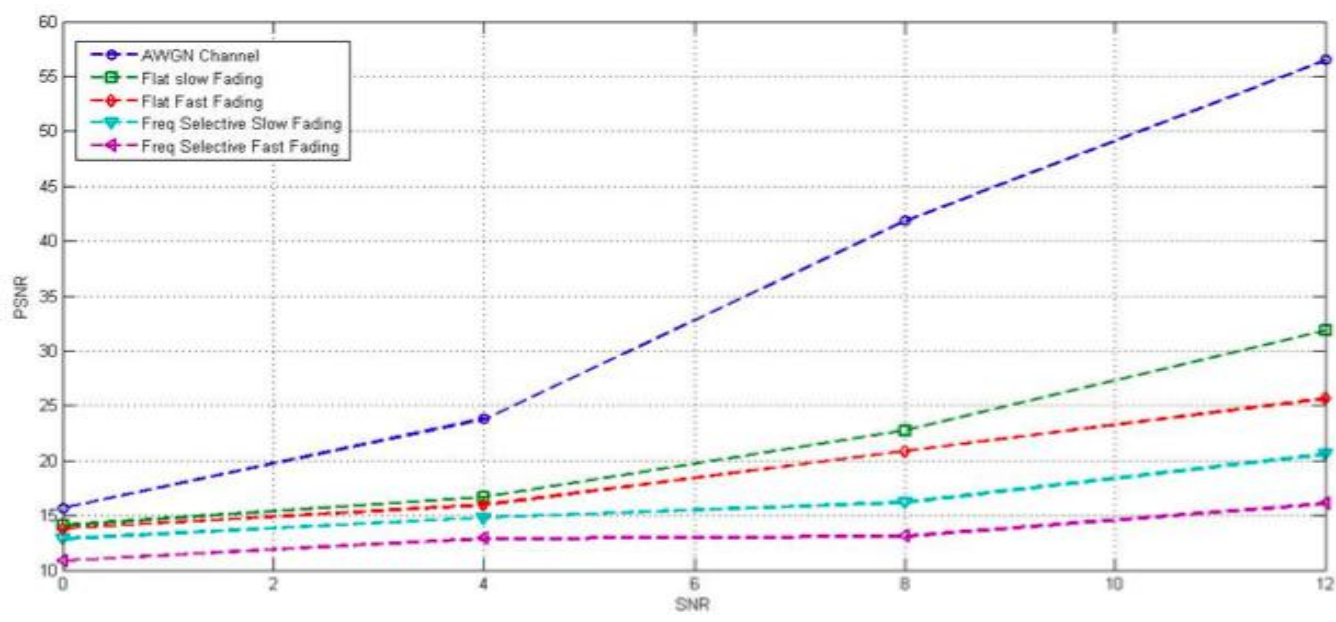

Figure 9 Comparing PSNR of The Different Channels

\section{CONCLUSION}

OFDM systems are very efficient in handling bad conditions and high data rates. Fading channels, however, are very common in wireless communications. They affect the process of signal reception after causing losses in transmitted signal. These effects that fading channels have on the performance of OFDM systems are investigated. MATLAB was used to simulate wireless fading channels that are ether based on Doppler spread or Delay Spread. Quality of received images, BER, PSNRs is the aspects used to evaluate the extent of these effects. In conclusion, fading that is caused by delay spread caused less distortion than fading that result from Doppler spread. We found that Distortion to the received signal is higher when the speed of the receiver is increased.

\section{REFRENCES}

[1] BBC Research Department, E. D. An introduction to digital modulation and OFDM techniques, 1993.

[2] Y. Cho, J. Kim, W. Yan, \& C. Kang, MIMO-OFDM wireless communications with MATLAB. (Singapore: Wiley, 2010).

[3] R. Frederiksen, F. Prasad, An overview of OFDM and related techniques towards development of future wireless multimedia communications, IEEE Radio and Wireless Conference Proceedings, 2002.

[4] W. Spring, \& T. Mathur, Small scale fading in radio propagation, 2005.

[5] T. Rappaport. Wireless Communication. (Upper Saddle River, NJ: Prentice Hall, 1996).

[6] J. C. Chuang, The effects of time delay spread on portable radio communications channels with digital modulation, IEEE journal on selected areas in communications, 1987.

[7] V. Jeyasri, Mobile communications, India: Technical Publications Pune, 2009.

[8] M. Engels. Wireless OFDM systems 2 (Boston: KLUWE Academic, 2002).

[9] S. Gayatri, P. Prabhu, \& M, Shankar, Simulation of flat fading using MATLAB for classroom instruction. IEEE TRANSACTIONS ON EDUCATION.

[10] K, SANKAR. Understanding an OFDM transmission, 2008. Retrieved April 15, 2012, from http://www.dsplog.com/2008/02/03/understanding-an-ofdm-transmission

[11] K. Lee, H. Lee, N. Chang, and J. Kang, Design of novel orthogonal space-time-frequency block codes for OFDM systems Over Fading Channel Environments, Proc. IEEE 66th Vehicular Technology Conference, 2007, pp. 521525 . 